\title{
Soluble Fms-like Tyrosine Kinase 1 Is a Novel Predictor of Brain Natriuretic Peptide Elevation
}

\section{Results of a 5-year Observational Study Involving Japanese Patients With Coronary Artery Disease}

\author{
Ryo Kameda, ${ }^{1}$ MD, Minako Yamaoka-Tojo, ${ }^{1,2}$ MD, Akihiro Makino, ${ }^{1}$ BSc, Kazuki WaKaume, ${ }^{1}$ BSc, \\ Shinji Nemoto, ${ }^{1}$ BSc, Lisa Kitasato, ${ }^{1}$ MD, Takao Shimohama, ${ }^{1,3}$ MD, Taiki Tojo, ${ }^{1,3}$ MD, \\ Yoji MaCHIDA, ${ }^{3} \mathrm{MD}$, and Tohru IzUmI, ${ }^{1,3} \mathrm{MD}$
}

\begin{abstract}
SUMMARY
Soluble fms-like tyrosine kinase 1 (sFlt-1) is an endogenous inhibitor of vascular endothelial growth factor, which is involved in cardiovascular remodeling and atherosclerosis development. To examine the predictive role of sFlt-1 levels in patients with asymptomatic heart failure, we measured circulating sFlt-1 in patients with or without coronary artery disease (CAD). We analyzed 88 Japanese patients with CAD or patients at high risk for atherosclerosis and who were undergoing total risk management for cardiovascular disease prevention. Circulating sFlt-1 levels correlated with the increase in plasma brain natriuretic peptide levels $(\triangle \mathrm{BNP})$ from baseline to the observed levels 5 years later in CAD patients, patients with previous myocardial infarction, and men. $\triangle \mathrm{BNP}$ levels correlated with sFlt-1 levels in the high-sFlt-1 patients with $\mathrm{CAD}(r=0.511, P<0.01)$. In all patients, end-systolic volume index $(\triangle \mathrm{ESVI})$ increased in correlation with a decrease in left ventricular ejection fraction $(\Delta \mathrm{EF})$ in the long-term observation, independent of their history of myocardial infarction $\left(\triangle \mathrm{ESVI}=2.5 \mathrm{~mL} / \mathrm{m}^{2}\right.$ increase/year). Baseline level of sFlt-1 was independent of $\triangle \mathrm{ESVI}$ or $\triangle \mathrm{EF}$. The present 5-year observational study demonstrated that high sFlt-1 levels predicted moderate increases in BNP levels in $\mathrm{CAD}$ patients. Moreover, $\triangle \mathrm{BNP}$ was correlated with $\triangle \mathrm{ESVI} /$ year in CAD patients with high-sFlt-1 levels. These data suggest that high sFlt-1 levels may be an effective biomarker to predict the progression of heart failure in patients with CAD. (Int Heart J 2013; 54: 133-139)
\end{abstract}

Key words: Coronary artery disease, Growth factor, Heart failure, Cardiac remodeling, Biomarker, Preventive cardiology

$\mathrm{P}$ atients with heart failure usually develop symptoms gradually, and they become increasingly less active and experience more frequent episodes of acute heart failure, known as decompensation. ${ }^{1)}$ The prevalence of heart failure, worldwide, continues to increase, with high rates of associated morbidity and mortality, imposing enormous human, social, and economic costs. Heart failure occurs predominantly because of coronary artery disease (CAD). Whether caused by myocardial infarction (MI) or chronic pressure-overload, like hypertensive heart disease or aortic valve stenosis, cardiac remodeling develops in response to cardiac injury or abnormal hemodynamic stress. ${ }^{2}$ Cardiac remodeling is characterized by cardiac hypertrophy, contractile dysfunction, matrix metalloproteinase accumulation, increased cardiomyocyte apoptosis, and abnormal myocardial energetics. ${ }^{3-5)}$ Pathological left ventricular (LV) remodeling is thought to be closely linked to the activation of a series of neurohumoral factors, which are up- regulated after myocardial injury and in the setting of increased LV wall stress and hemodynamic derangement. $\left.{ }^{6}\right)$

Fms-like tyrosine kinase 1 (Flt-1) is a receptor of tyrosine kinase and a member of the vascular endothelial growth factor (VEGF) receptor family. ${ }^{7)}$ Soluble Flt-1 (sFlt-1) is an endogenous inhibitor of VEGF-A and placental growth factor (PIGF), which are thought to exacerbate atherosclerosis. ${ }^{8)}$ sFlt1 is generated by the alternative splicing of the Flt- 1 gene in the vascular endothelium and placenta. ${ }^{9,10)} \mathrm{PIGF} / \mathrm{sFlt}-1$ levels have been reported to be positively associated with the presence of CAD and the angiographic severity of CAD. ${ }^{11)}$ In patients with chest pain and acute coronary syndrome, higher PlGF levels have been observed in those with MI and are associated with an increased risk of short- and long-term adverse outcomes. ${ }^{12-14)}$ There are conflicting results regarding circulating sFlt-1 levels in patients with CAD, with some studies noting higher levels during acute MI compared with control pa-

From the ${ }^{1}$ Kitasato University Graduate School of Medical Sciences, ${ }^{2}$ Department of Rehabilitation, Kitasato University School of Allied Health Sciences, and ${ }^{3}$ Department of Cardioangiology, Kitasato University School of Medicine, Kanagawa, Japan.

This work was partly supposed by KAKENHI: Health Labor Science Research Grant 10103153 (TI); Grants-in-Aid for Young Scientists 21790743 (MY-T) and 21790744 (TT) from the Ministry of Education, Culture, Sports, Science and Technology of Japan; Grant-in Aid for Research Project, No. 2012-0000 from Kitasato University School of Allied Health Sciences (MY-T); and Grant for Research Project from Kitasato University School of Medical Sciences, Sagamihara, Japan (MY-T).

Address for correspondence: Minako Yamaoka-Tojo, MD, Department of Rehabilitation, Kitasato University School of Allied Health Sciences, 1-15-1 Kitasato, Minami-ku, Sagamihara, Kanagawa 252-0373, Japan.

Received for publication November 20, 2012.

Revised and accepted January 28, 2013. 
tients, ${ }^{15)}$ and others showing lower plasma sFlt-1 levels in patients during the acute phase of MI compared with control subjects. ${ }^{16,17)}$ Although PIGF and sFlt-1 might be important biomarkers of chronic heart failure, neither factor has been fully studied in patients with heart failure. Circulating PlGF was measured in a cross-sectional study of 98 patients, in which there was a positive relationship between PIGF levels and the New York Heart Association (NYHA) functional class of ischemic heart failure patients. ${ }^{18)}$

On the other hand, a few experimental and cross-sectional clinical studies have attempted to evaluate the association between sFlt-1 and CAD. Because of its potent effects on cardiac hypertrophy, angiogenesis, and atherogenesis, we hypothesized that circulating sFlt-1 levels would be associated with LV remodeling in patients with CAD. The aim of this study was to examine whether the circulating sFlt-1 level is a biomarker predictive of cardiac remodeling in $\mathrm{CAD}$ patients and patients at high risk for CAD.

\section{Methods}

Study population: The study included 88 Japanese out-patients who were undergoing total risk management for the secondary prevention of cardiovascular disease as part of the Kitasato Registry for Cardiovascular Disease Prevention (KRCDP) program. The patients either had a diagnosis of CAD or were at high risk of developing CAD. We defined coronary artery disease as previous myocardial infarction and angina pectoris with medical treatment and/or coronary intervention. High risk patients were defined as having two or more coronary artery risk factors: diabetes mellitus, hypertension, dyslipidemia, and obesity. The follow-up period was 5 years for all patients. All subjects gave informed consent before participating in the study, and the Ethics Committee of Kitasato University Hospital approved the study design.

The exclusion criteria were liver (serum aminotransferase, $>2 \times$ the upper limit of normal) or severe kidney dysfunction (eGFR $\left.<30 \mathrm{~mL} \cdot \mathrm{min}^{-1} \cdot 1.73 \mathrm{~m}^{-2} \mathrm{mg} / \mathrm{dL}\right)$, a history of inflammatory disease, or malignant neoplasm.

Measurement of biomarkers: Blood samples were collected by venipuncture after an overnight fast. Biochemical markers such as triglycerides, low-density lipoprotein (LDL) cholesterol, high-density lipoprotein (HDL) cholesterol, insulin, plasma glucose, glycated hemoglobin (HbA1c), uric acid, and brain natriuretic peptide (BNP) levels were measured.

Circulating levels of human sFlt-1 were determined by enzyme-linked immunosorbent assay (ELISA) using a Human sVEGF R1/Flt-1 Immunoassay Quantikine ELISA (R\&D Systems, Minneapolis, MN, USA) according to the manufacturer's assay procedure.

Clinical parameters: The clinical parameters assessed included age, gender, and coronary risk factors (hypertension, diabetes mellitus [DM], and dyslipidemia). The diagnostic criteria for the coronary risk factors were as follows: hypertension, blood pressure more than 140/90 $\mathrm{mmHg}$, and/or a history of taking antihypertensive medications; diabetes mellitus, as defined by a fasting plasma glucose level $>126 \mathrm{mg} / \mathrm{dL}$, or a casual plasma glucose level > $200 \mathrm{mg} / \mathrm{dL}$; dyslipidemia, as defined by serum total cholesterol levels of $>220 \mathrm{mg} / \mathrm{dL}$ or serum triglyceride levels of $>150 \mathrm{mg} / \mathrm{dL}$ or LDL-cholesterol levels of $>140 \mathrm{mg} / \mathrm{dL}$. Angiographically defined patients and those with a history of coronary intervention were included in the CAD group.

Perfusion SPECT: Based on electrocardiography-gated single-photon emission computed tomography (SPECT), left ventricular function, including ventricular volumes, systolic and diastolic functions, and systolic wall thickening, were analyzed using common nuclear cardiology software. At the time of registration in the KRCDP program and at every annual visit, myocardial perfusion SPECT was performed according to a standard protocol in most CAD patients.

Statistical analysis: Continuous data are summarized as the mean $\pm \mathrm{SD}$, median (interquartile range), and categorical data are expressed as percentages. Statistical analyses were performed by one-way analysis of variance (ANOVA) or by 2-tailed $t$ test. To evaluate correlations between $\triangle \mathrm{BNP}$ and selected variables, Spearman's correlation coefficients between variables were calculated. To assess whether sFlt-1 was an independent predictor of $\triangle \mathrm{BNP}$, multivariable logistic regression models were constructed. JMP statistical software 9.0 (SAS Institute, Cary, NC, USA) was used. A $P$ value of $<0.05$ was considered statistically significant.

\section{RESULTS}

Clinical characteristics during the 5-year observation period: Eighty-eight CAD patients and patients at high risk for CAD participated in the 5-year observational study (average age, 64.9 years; women, $27.3 \%$ ). As shown in Table I, $65.9 \%$ of the patients had CAD (including patients with previous MI), $59.1 \%$ had hypertension, $70.5 \%$ had dyslipidemia, $28.4 \%$ had diabetes mellitus, and $35.2 \%$ had a history of a previous MI. The mean baseline level of serum sFlt-1 was $189.6 \pm 95.5 \mathrm{ng} /$ $\mathrm{mL}$ in all subjects (Table I). At the conclusion of the 5-year disease management program, the circulating levels of sFlt-1 were significantly lower in all patients $(P<0.01)$. Only circulating levels of LDL were significantly lowered by cholesterol lowering diet therapy education during the 5-year disease management period $(P<0.01)$. Of the 88 participants, 2 were given additional statin therapy during the study period. There was no correlation between statin use and a decrease in the circulating levels of sFlt-1. No other clinical data, including body mass index (BMI), triglycerides, HbA1c, and BNP levels, were changed in these patients undergoing 5-year total risk management for cardiovascular disease prevention in KRCDP.

Circulating levels of sFlt-1 in CAD patients: Circulating levels of sFlt-1 were higher in men $(200.7 \pm 108.0 \mathrm{ng} / \mathrm{mL})$ compared to women $(160.1 \pm 34.3 \mathrm{ng} / \mathrm{mL} ; P<0.01)$. Among the elderly patients ( $>65$ years-old), sFlt-1 levels were different between men $(222.5 \pm 143.7 \mathrm{ng} / \mathrm{mL})$ and women $(160.2 \pm 38.3 \mathrm{ng} / \mathrm{mL}$; $P=0.033$ ). As shown in Figure 1, sFlt-1 levels were significantly higher in patients with CAD compared to those without CAD (127\% increase; $P<0.01)$. Moreover, circulating sFlt-1 concentrations were, on average, $140 \%$ higher in old MI (OMI) patients compared to non-MI patients $(P<0.01)$. When the patients were divided into 2 groups, based on circulating sFlt-1 levels, OMI and baseline estimated glomerular filtration rates (eGFRs) were significantly different between the 2 groups (Table III).

Association between sFlt-1 and coronary risk factors: There 
Table I. Participant Characteristics $(n=88)$

\begin{tabular}{|c|c|c|c|}
\hline & Baseline & 5 years & $P$ \\
\hline Age (years) & $64.9 \pm 9.4$ & & \\
\hline Sex, female $(\%)$ & $24(27.3)$ & & \\
\hline $\mathrm{BMI}\left(\mathrm{kg} / \mathrm{m}^{2}\right)$ & $24.5 \pm 3.1$ & $23.8 \pm 3.2$ & NS \\
\hline Coronary artery disease, $n(\%)$ & $58(65.9)$ & & \\
\hline Previous history of OMI, $n(\%)$ & $31(35.2)$ & & \\
\hline Diabetes mellitus, $n(\%)$ & $25(28.4)$ & & \\
\hline Hypertension, $n(\%)$ & $52(59.1)$ & & \\
\hline Dyslipidemia, $n(\%)$ & $62(70.5)$ & & \\
\hline LDL cholesterol (mg/dL) & $133.2 \pm 46.1$ & $113.3 \pm 27.4$ & $<0.01$ \\
\hline HDL cholesterol (mg/dL) & $54.1 \pm 18.0$ & $56.2 \pm 20.3$ & NS \\
\hline Triglycerides (mg/dL) & $131.0 \pm 82.4$ & $125.4 \pm 89.0$ & NS \\
\hline $\operatorname{HbAlc}(\%)$ & $5.5 \pm 0.6$ & $5.6 \pm 0.7$ & NS \\
\hline $\mathrm{BNP}(\mathrm{pg} / \mathrm{mL})$ & $45.6 \pm 48.1$ & $62.4 \pm 108.7$ & NS \\
\hline $\mathrm{eGFR}\left(\mathrm{mL} \cdot \mathrm{min}^{-1} \cdot 1.73 \mathrm{~m}^{-2}\right)$ & $72.0(37.8-135.3)$ & $70.4(36.0-120.1)$ & $<0.05$ \\
\hline $\mathrm{PWV}(\mathrm{m} / \mathrm{s})$ & $1544.1 \pm(1041-2560)$ & $1560.2(1075-2227)$ & NS \\
\hline sFlt-1 (pg/mL) & $189.6 \pm 95.5$ & $71.3 \pm 20.2$ & $<0.01$ \\
\hline Statin use, $n(\%)$ & $37(42.0 \%)$ & $39(44.3 \%)$ & NS \\
\hline$\beta$-Blockers, $n(\%)$ & $35(39.8 \%)$ & & \\
\hline RAAS inhibitors, $n(\%)$ & $30(34.1 \%)$ & & \\
\hline Ca-channel blockers, $n(\%)$ & $49(55.7 \%)$ & & \\
\hline Aspirin, $n(\%)$ & $54(61.4 \%)$ & & \\
\hline
\end{tabular}

Continuous variables are presented as the mean $\pm \mathrm{SD}$, median (interquartile range), whereas categorical variables are presented as counts and percentages. BMI indicates body mass index; OMI, old myocardial infarction; LDL, low density lipoprotein; HDL, high density lipoprotein; HbA1c, glycated hemoglobin; BNP, brain natriuretic peptide; eGFR, estimated glomerular filtration rate; PWV, pulse wave velocity; sFlt-1, soluble Fms-like tyrosine kinase 1; RAAS, renin-angiotensin-aldosterone system; Ca-channel, calcium channel; and NS, not significant.

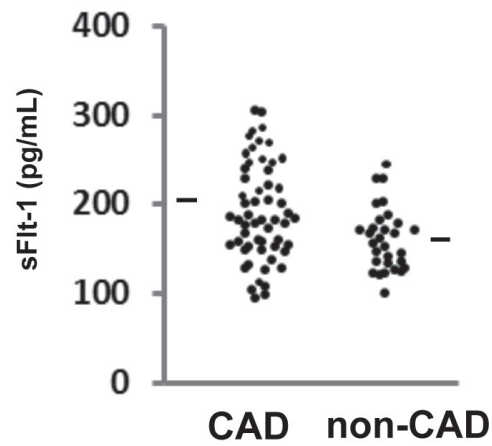

Figure 1. Circulating levels of soluble fms-like tyrosine kinase 1 (sFlt-1), at baseline, in patients with and without coronary artery disease (CAD). Scatter plot showing the sFlt-1 $(\mathrm{ng} / \mathrm{mL})$ levels in CAD patients $(n=58)$ and patients at high risk of $\mathrm{CAD}(n=30)$. The bars indicate the mean values.

was no significant relationship between circulating levels of sFlt-1 and any coronary risk factors, including DM, hypertension, dyslipidemia, age, or sex (Table III). Patient groups were also compared on the basis of their number of coronary risk factors (DM, hypertension, dyslipidemia, chronic kidney disease (CKD), and obesity [BMI $\left.\geq 30 \mathrm{~kg} / \mathrm{m}^{2}\right]$ ). Serum sFlt-1 and PlGF levels were not significantly correlated with any of the coronary risk factors nor with an accumulation of coronary risk factors.

Association of serum sFlt-1 with $\triangle \mathrm{BNP}$ : In all patients, neither the baseline sFlt-1 levels nor an increase in plasma BNP levels $(\triangle \mathrm{BNP})$ from baseline to 5 years later was significantly correlated within the study cohort. Baseline levels of sFlt-1 were correlated with $\triangle \mathrm{BNP}$ from baseline to 5 years later in CAD patients $(r=0.303, P<0.05)$, in men $(r=0.295, P<0.05)$, and in those with previous MI $(r=0.558, P<0.01)$, hypertension $(r=0.291, P<0.05)$, and dyslipidemia $(r=0.311, P<$ $0.05)$. Markedly, there was no significant association between sFlt- 1 levels and $\triangle \mathrm{BNP}$ in patients with DM $(r=0.179, P=$ 0.393).

Cardiac remodeling in CAD patients: Because of a weak but significantly positive correlation between circulating sFlt-1 levels and $\triangle \mathrm{BNP}$ in $\mathrm{CAD}$ patients, we examined the changes in cardiac remodeling, as measured by nuclear perfusion imaging, in these patients. In CAD patients, $72.4 \%$ were examined by myocardial perfusion SPECT. We chose $\triangle \mathrm{ESVI} /$ year, as detected by rest SPECT, as an indicator of cardiac remodeling. As shown in Figure 2A, $\triangle$ ESVI/year had a positive correlation to $\triangle \mathrm{BNP}$ in CAD patients $(r=0.379, P<0.05)$. $\triangle \mathrm{BNP}$ values were also strongly correlated with $\triangle \mathrm{ESVI} /$ year in high-sFlt-1 patients with CAD, independent of history of $\mathrm{MI}(\triangle \mathrm{ESVI}=2.5$ $\pm 3.3 \mathrm{~mL} / \mathrm{m}^{2}$ increase/year, decrease in ejection fraction $[\Delta \mathrm{EF}]$ $=2.4 \% \pm 6.4 \%$ decrease/year) (Table IV). In addition, $\Delta \mathrm{ESVI} /$ year was inversely correlated with $\triangle \mathrm{EF} /$ year in $\mathrm{CAD}$ patients (Figure 2B). Importantly, $\triangle \mathrm{ESVI}$ increased in the long-term observation, independent of their history of myocardial infarction $\left(\triangle \mathrm{ESVI}=2.5 \mathrm{~mL} / \mathrm{m}^{2}\right.$ increase/year). However, the baseline level of sFlt-1 was independent of $\triangle \mathrm{ESVI}$ or $\Delta \mathrm{EF}$.

sFlt-1 as a predictive biomarker: The patients were classified into 2 groups based on whether they had a baseline diagnosis of CAD. There was a significant difference between the 2 groups with respect to HbA1c, eGFR, sFlt-1, sFlt-1/PlGF, and the presence of hypertension (Table II). In the high-sFlt-1 group, $\triangle \mathrm{BNP}$ levels were correlated with sFlt-1 $(r=0.456, P<$ $0.01)$, especially in CAD patients $(n=44, r=0.511, P<0.01)$, but not in the low-sFlt-1 group $(n=44, r=-0.237, P=0.381)$. As shown in Table IV, $\triangle \mathrm{BNP}$ levels were significantly higher 
Table II. Demographic and Clinical Characteristics of CAD Patients Divided Into 2 Groups, With Baseline sFlt-1 Levels

\begin{tabular}{|c|c|c|c|}
\hline & $\begin{array}{c}\text { High-sFlt-1 } \\
n=44\end{array}$ & $\begin{array}{c}\text { Low-sFlt-1 } \\
n=44\end{array}$ & $P$ \\
\hline Age (years) & $66.6 \pm 10.4$ & $63.1 \pm 8.1$ & NS \\
\hline Sex, female $(\%)$ & $9(20.5)$ & $15(34.1)$ & NS \\
\hline BMI $\left(\mathrm{kg} / \mathrm{m}^{2}\right)$ & $24.6 \pm 3.3$ & $24.3 \pm 2.6$ & NS \\
\hline Previous history of OMI, $n(\%)$ & $23(52.3)$ & $8(18.2)$ & $<0.01$ \\
\hline Diabetes mellitus, $n(\%)$ & $11(25.0)$ & $14(31.8)$ & NS \\
\hline Hypertension, $n(\%)$ & $25(56.8)$ & $27(61.4)$ & NS \\
\hline Dyslipidemia, $n(\%)$ & $33(75.0)$ & $29(65.9)$ & NS \\
\hline LDL cholesterol (mg/dL) & $133.3 \pm 41.0$ & $133.0 \pm 51.3$ & NS \\
\hline HDL cholesterol (mg/dL) & $53.3 \pm 11.5$ & $55.0 \pm 22.8$ & NS \\
\hline Triglyceride (mg/dL) & $130.3 \pm 89.0$ & $131.8 \pm 76.3$ & NS \\
\hline $\mathrm{HbAlc}(\%)$ & $5.5 \pm 0.7$ & $5.5 \pm 0.5$ & NS \\
\hline Baseline BNP (pg/mL) & $49.2 \pm 43.1$ & $35.9 \pm 52.2$ & NS \\
\hline 5-year BNP (pg/mL) & $71.7 \pm 89.3$ & $34.6 \pm 29.6$ & $<0.05$ \\
\hline$\Delta \mathrm{BNP}(\mathrm{pg} / \mathrm{mL})$ & $51.6 \pm 103.1$ & $38.7 \pm 100.9$ & NS \\
\hline Baseline eGFR $\left(\mathrm{mL} \cdot \mathrm{min}^{-1} \cdot 1.73 \mathrm{~m}^{-2}\right)$ & $72.7(37.9-113.2)$ & $70.1(40.5-135.3)$ & $<0.05$ \\
\hline 5 -year eGFR $\left(\mathrm{mL} \cdot \mathrm{min}^{-1} \cdot 1.73 \mathrm{~m}^{-2}\right)$ & $66.3(36.0-98.6)$ & $71.7(37.8-120.2)$ & NS \\
\hline $\mathrm{PWV}(\mathrm{m} / \mathrm{s})$ & $1547.3(1041-2560)$ & $1544(1124-2250)$ & NS \\
\hline sFlt-1 (pg/mL) & $238.4 \pm 115.4$ & $140.8 \pm 21.6$ & $<0.01$ \\
\hline PlGF (pg/mL) & $52.7 \pm 14.7$ & $59.4 \pm 15.4$ & $<0.05$ \\
\hline 5-year sFlt-1 (pg/mL) & $75.4 \pm 21.7$ & $67.1 \pm 17.8$ & NS \\
\hline$\Delta$ sFlt-1 (pg/mL) & $-65.2 \pm 12.2$ & $-51.8 \pm 16.1$ & NS \\
\hline sFlt-1/PlGF & $5.0 \pm 2.9$ & $2.6 \pm 1.0$ & $<0.01$ \\
\hline
\end{tabular}

Continuous variables are presented as the mean $\pm \mathrm{SD}$, median (interquartile range), whereas categorical variables are presented as counts and percentages. CAD indicates coronary artery disease; BMI, body mass index; OMI, old myocardial infarction; LDL, low density lipoprotein; HDL, high density lipoprotein; HbAlc, glycated hemoglobin; BNP, brain natriuretic peptide; eGFR, estimated glomerular filtration rate; PWV, pulse wave velocity; sFlt-1, soluble fms-like tyrosine kinase 1; PIGF, placental growth factor; and NS, not significant.
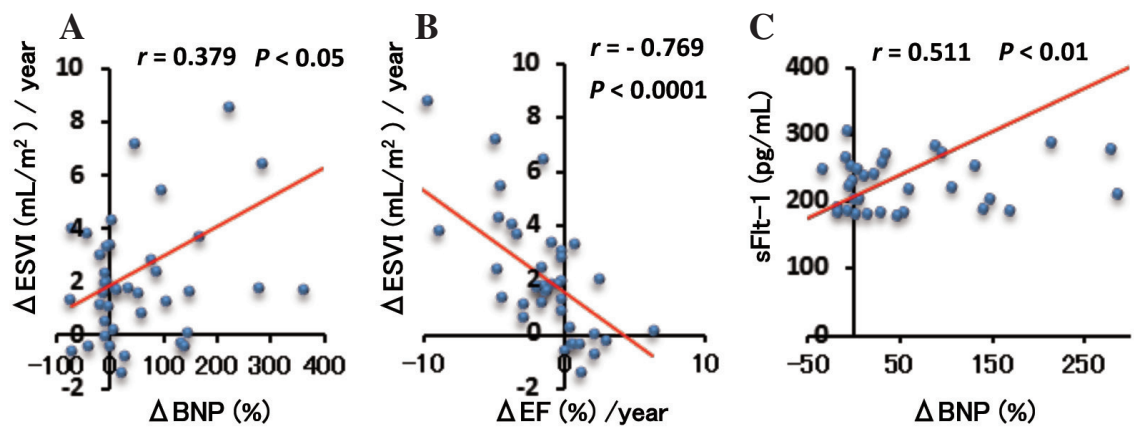

Figure 2. Associations between changes in brain natriuretic peptide ( $\triangle \mathrm{BNP})$, soluble fms-like tyrosine kinase 1 (sFlt-1), cardiac remodeling, and its function. In 42 patients with coronary artery disease (CAD), changes in cardiac remodeling over a 5-year period were measured by nuclear myocardial perfusion single-photon emission computed tomography. Increasing levels of plasma BNP $(\triangle \mathrm{BNP})$ during the 5-year period were correlated with annual increases in the end-systolic volume index ( $\triangle \mathrm{ESVI} / \mathrm{year})$ in the left ventricle of patients with $\mathrm{CAD}(\mathbf{A}) . \triangle \mathrm{ESVI} / \mathrm{year}$ was correlated with an annual decrease in ejection fraction $(\triangle \mathrm{EF} / \mathrm{year})$ in $\mathrm{CAD}$ patients (B). $\triangle \mathrm{BNP}$ was significantly correlated with sFlt-1 levels in CAD patients in the high-sFlt-1 group (C).

in the high-sFlt-1 group compared to those in the low-sFlt-1 group $(P<0.05)$. Multivariate logistic regression analyses, adjusted for age and sex, identified circulating sFlt-1 level as an independent predictor of BNP progression (Table V). As shown in Figure 2C, sFlt-1 levels could be a biomarker, predicting BNP elevations 5 years later $(P<0.01)$.

\section{Discussion}

The present study is the first to report that high levels of sFlt-1 in patients with CAD are associated with BNP level in- creases and LV remodeling 5 years later. Our results also showed that high sFlt-1 levels were independent predictors of moderate levels of BNP elevation, which may reflect the condition of asymptomatic heart failure, and ACC/AHA Stages A and $\mathrm{B}$ heart failure. These data suggest that high sFlt-1 levels may be an effective biomarker for predicting the progression of heart failure in patients with CAD. Furthermore, as shown in Table II and Table III, both the 5 year sFlt- 1 levels and the decrease in plasma sFlt-1 levels $(\Delta$ sFlt- 1$)$ from baseline to 5 years later were not significantly different between the CAD group and non-CAD group, high-sFlt-1 group and low-sFlt-1 group. However, $\Delta$ sFlt-1 levels may reflect BNP progression 
Table III. Cardiac Remodeling Detected by Rest Myocardial Perfusion Single-photon Emission Computed Tomography in CAD Patients $(n=42)$

\begin{tabular}{|c|c|c|c|c|}
\hline & Sampling point & $\mathrm{CAD}(n=42)$ & High sFlt-1 $(n=21)$ & Low-sFlt-1 $(n=21)$ \\
\hline Age (years) & Baseline & $67.2 \pm 8.2$ & $67.1 \pm 8.2$ & $67.3 \pm 8.3$ \\
\hline Sex, female $(\%)$ & Baseline & $10(23.8)$ & $2(9.5)$ & $8(38.1)^{*}$ \\
\hline BMI $\left(\mathrm{kg} / \mathrm{m}^{2}\right)$ & Baseline & $24.1 \pm 3.8$ & $23.4 \pm 2.8$ & $24.9 \pm 4.6$ \\
\hline Previous history of OMI, $n(\%)$ & Baseline & $22(52.4)$ & $12(57.1)$ & $10(47.6)$ \\
\hline sFlt-1 (pg/mL) & Baseline & $189.9 \pm 54.3$ & $234.2 \pm 39.4$ & $147.7 \pm 24.3^{*}$ \\
\hline \multirow[t]{2}{*}{$\mathrm{BNP}(\mathrm{pg} / \mathrm{mL})$} & Baseline & $37.4 \pm 50.9$ & $32.2 \pm 25.7$ & $42.6 \pm 67.7$ \\
\hline & 5 years later & $53.6 \pm 77.4$ & $73.4 \pm 104.4$ & $66.2 \pm 105.5$ \\
\hline$\Delta \mathrm{BNP}(\% /$ year $)$ & & $18.0 \pm 40.5$ & $31.3 \pm 52.8$ & $4.7 \pm 14.4^{*}$ \\
\hline \multirow[t]{2}{*}{$\operatorname{EDVI}\left(\mathrm{mL} / \mathrm{m}^{2}\right)$} & Baseline & $49.8 \pm 19.1$ & $53.8 \pm 21.1$ & $46.0 \pm 16.7$ \\
\hline & 5 years later & $57.8 \pm 19.1$ & $61.9 \pm 20.6$ & $53.8 \pm 17.0$ \\
\hline$\Delta$ EDVI/year $\left(\mathrm{mL} \cdot \mathrm{m}^{-2} \cdot\right.$ year $\left.^{-1}\right)$ & & $3.5 \pm 4.1$ & $2.9 \pm 3.5$ & $4.1 \pm 4.5$ \\
\hline \multirow[t]{2}{*}{$\operatorname{ESVI}\left(\mathrm{mL} / \mathrm{m}^{2}\right)$} & Baseline & $19.8 \pm 15.6$ & $23.1 \pm 17.0$ & $16.7 \pm 13.9$ \\
\hline & 5 years later & $24.6 \pm 16.3$ & $27.4 \pm 16.7$ & $22.0 \pm 15.9$ \\
\hline$\Delta$ ESVI/year $\left(\mathrm{mL} \cdot \mathrm{m}^{-2} \cdot\right.$ year $\left.^{-1}\right)$ & & $2.5 \pm 3.3$ & $1.6 \pm 1.8$ & $3.4 \pm 4.1$ \\
\hline \multirow[t]{2}{*}{$\mathrm{EF}(\%)$} & Baseline & $63.4 \pm 13.8$ & $60.6 \pm 13.1$ & $67.5 \pm 13.9$ \\
\hline & 5 years later & $60.4 \pm 13.2$ & $58.8 \pm 11.3$ & $62.0 \pm 14.9$ \\
\hline$\Delta \mathrm{EF} /$ year (\% decrease/year) & & $-2.4 \pm 6.4$ & $-0.6 \pm 2.0$ & $-4.2 \pm 8.4$ \\
\hline
\end{tabular}

${ }^{*} P<0.05$. Data are presented the mean \pm SD. CAD indicates coronary artery disease; OMI, old myocardial infarction; sFlt1, soluble fms-like tyrosine kinase 1; BNP, brain natriuretic peptide; EDVI, end-diastolic volume index; ESVI, end-systolic volume index; and $\mathrm{EF}$, ejection fraction.

Table IV. Demographic and Clinical Characteristics of CAD Patients $(n=88)$

\begin{tabular}{lccc}
\hline & CAD $(+) n=58$ & CAD $(-) n=30$ & $P$ \\
\hline Age (years) & $64.7 \pm 9.4$ & $65.2 \pm 9.6$ & NS \\
Sex, female $(\%)$ & $13(22.4)$ & $11(36.7)$ & NS \\
BMI $\left(\mathrm{kg} / \mathrm{m}^{2}\right)$ & $24.6 \pm 3.3$ & $24.3 \pm 2.6$ & $\mathrm{NS}$ \\
Previous history of OMI, $n(\%)$ & $31(53.4)$ & $0(0.0)$ & \\
Diabetes mellitus, $n(\%)$ & $18(31.0)$ & $7(23.3)$ & $\mathrm{NS}$ \\
Hypertension, $n(\%)$ & $27(46.6)$ & $25(83.3)$ & $<0.01$ \\
Dyslipidemia, $n(\%)$ & $43(74.1)$ & $19(63.3)$ & $\mathrm{NS}$ \\
LDL cholesterol $(\mathrm{mg} / \mathrm{dL})$ & $139.4 \pm 48.0$ & $121.2 \pm 40.6$ & $\mathrm{NS}$ \\
HDL cholesterol $(\mathrm{mg} / \mathrm{dL})$ & $51.7 \pm 11.0$ & $58.8 \pm 26.4$ & $\mathrm{NS}$ \\
Triglycerides $(\mathrm{mg} / \mathrm{dL})$ & $133.5 \pm 86.2$ & $126.3 \pm 75.8$ & $\mathrm{NS}$ \\
HbAlc $(\%)$ & $5.6 \pm 0.6$ & $5.3 \pm 0.4$ & $<0.05$ \\
Baseline BNP $(\mathrm{pg} / \mathrm{mL})$ & $43.6 \pm 50.2$ & $40.5 \pm 44.8$ & $\mathrm{NS}$ \\
5-year BNP $(\mathrm{pg} / \mathrm{mL})$ & $59.2 \pm 78.3$ & $41.5 \pm 43.7$ & $\mathrm{NS}$ \\
$\Delta \mathrm{BNP}(\mathrm{pg} / \mathrm{mL})$ & $54.4 \pm 112.3$ & $27.2 \pm 75.4$ & $\mathrm{NS}$ \\
eGFR $\left(\mathrm{mL} \cdot \mathrm{min}{ }^{-1} \cdot 1.73 \mathrm{~m} \mathrm{~m}^{-2}\right)$ & $66.2(37.9-135.3)$ & $78.2(46.2-115.2)$ & $<0.05$ \\
PWV $(\mathrm{m} / \mathrm{s})$ & $1540.5(1041-2250)$ & $1573.3(1439-1682)$ & $\mathrm{NS}$ \\
sFlt-1 $(\mathrm{pg} / \mathrm{mL})$ & $204.5 \pm 113.0$ & $160.8 \pm 35.3$ & $<0.01$ \\
5 -year sFlt-1 $(\mathrm{pg} / \mathrm{mL})$ & $71.2 \pm 19.9$ & $71.4 \pm 21.0$ & $\mathrm{NS}$ \\
$\Delta \mathrm{sFlt}-1(\mathrm{pg} / \mathrm{mL})$ & $-60.6 \pm 16.2$ & $-54.5 \pm 14.2$ & $\mathrm{NS}$ \\
PlGF $(\mathrm{pg} / \mathrm{mL})$ & $55.3 \pm 17.2$ & $57.5 \pm 11.1$ & $\mathrm{NS}$ \\
sFlt-1/PlGF & $4.2 \pm 2.8$ & $2.9 \pm 1.1$ & $<0.01$ \\
\hline
\end{tabular}

Continuous variables are presented as the mean $\pm \mathrm{SD}$, median (interquartile range), whereas categorical variables are presented as counts and percentages. CAD indicates coronary artery disease; BMI, body mass index; OMI, old myocardial infarction; LDL, low density lipoprotein; HDL, high density lipoprotein; HbAlc, glycated hemoglobin; BNP, brain natriuretic peptide; eGFR, estimated glomerular filtration rate; PWV, pulse wave velocity; sFlt-1, soluble fms-like tyrosine kinase 1; PIGF, placental growth factor; and NS, not significant.

because circulating levels of $\Delta$ sFlt-1 were higher in the CAD group and the high-sFlt-1 group compared to the non-CAD group and the low-sFlt-1 group. Although more data are needed, this finding may support the role of sFlt-1 levels as useful biomarkers of cardiac remodeling and predictors of clinical outcome.

Plasma BNP levels are known to be elevated in patients with symptomatic LV systolic dysfunction ${ }^{19,20)}$ and to correlate with NYHA class as well as prognosis. ${ }^{21,22)}$ In addition, irrespective of the degree of LV dysfunction, plasma BNP levels have been shown to be elevated in patients with various cardiac disorders, including previous MI, cardiomyopathy, valvular heart disease, hypertensive heart disease, and atrial fibrillation. ${ }^{19,23)}$ Therefore, plasma BNP levels may be a potential screening marker for identifying individuals with asymptomatic mild heart failure as well as those at high risk for moderateto-severe heart failure because of various forms of structural heart disease. $^{24)}$

The relationship between angiogenesis biomarkers and BNP levels has not been prospectively evaluated. In our study, 
Table V. Multiple Linear Regression to Assess the Variables Affecting the Increasing Value of BNP in All Patients $(n=88)$

\begin{tabular}{lcc}
\hline Variable & Univariate & Multivariate \\
\hline sFlt-1 $(\mathrm{pg} / \mathrm{mL})$ & 0.0088 & 0.0096 \\
Age (years) & 0.6039 & 0.8249 \\
Sex, male & 0.1510 & 0.3450 \\
LDL-C $(\mathrm{mg} / \mathrm{dL})$ & 0.3875 & - \\
$\mathrm{HDL}-\mathrm{C}(\mathrm{mg} / \mathrm{dL})$ & 0.8679 & - \\
$\mathrm{TG}(\mathrm{mg} / \mathrm{dL})$ & 0.7878 & - \\
$\mathrm{eGFR}\left(\mathrm{mL} \cdot \mathrm{min}^{-1} \cdot 1.73 \mathrm{~m}^{-2}\right)$ & 0.6738 & - \\
PWV $(\mathrm{m} / \mathrm{s})$ & 0.0949 & - \\
Diabetes mellitus & 0.7977 & - \\
Hypertension & 0.4891 & - \\
Number of risk factors & 0.3812 & - \\
Previous MI & 0.3812 & - \\
Presence of CAD & 0.2359 & - \\
\hline
\end{tabular}

If a patient was male or had the disease, then 1 was given as the variable; if not, then 0 was given as the variable. sFlt-1 indicates soluble fms-like tyrosine kinase 1; LDL-C, low-lipoprotein cholesterol; HDL-C, high-density lipoprotein cholesterol; TG, triglycerides; PWV, pulse wave velocity; eGFR, estimated glomerular filtration rate; MI, myocardial infarction; and $\mathrm{CAD}$, coronary artery disease.

higher sFlt-1 levels were strongly related to increases in BNP levels 5 years later. The clinical relevance and possible mechanism of our findings are discussed below. Studies in animal models suggest several potential mechanisms through which VEGF/Flt-1 signaling might modify the severity and course of heart failure. Circulating sFlt-1 opposes angiogenesis by binding to and sequestering salutary VEGF ligands in the circulation, resulting in endothelial dysfunction and vascular rarefaction that increases mechanical load on the heart. ${ }^{25,26)}$ Excess levels of sFlt-1 might also increase myocardial fibrosis and decrease myocardial capillary density, thereby directly affecting myocardial structure and function. ${ }^{27,28)}$

Although sFlt-1 levels were not associated with renal dysfunction in our study, sFlt-1 has been reported to impair glomerular function, possibly contributing to an unfavorable cardio-renal condition. In a previous study, Di Marco, et al also reported that sFlt-1 levels were independently associated with renal dysfunction. ${ }^{29)}$ In experimental animal models, exogenous administration of sFlt-1 to either pregnant or nonpregnant animals was found to induce widespread endothelial dysfunction, hypertension, and renal dysfunction. ${ }^{10,30)}$ These data suggest that the antiangiogenic effects of sFlt-1 may affect compensatory angiogenesis in diseased kidneys and failing hearts.

In the present study, the mechanism responsible for sFlt-1 elevation in patients with asymptomatic heart failure is not fully understood. Aldosterone is a steroid hormone that signals through the renal mineralocorticoid receptor (MR) to regulate blood pressure. ${ }^{31)}$ In clinical trials, aldosterone antagonists reduce cardiovascular ischemia and mortality by unknown mechanisms. ${ }^{32,33)}$ In mouse vessels with endothelial damage and human vessels from patients with atherosclerosis, aldosterone enhanced expression of PlGF and its receptor, Flt-1. ${ }^{34)}$ In atherosclerotic human vessels, MR antagonists were reported to inhibit PlGF expression. ${ }^{34)}$ In a mouse carotid wire injury model, aldosterone infusion accelerated vascular remodeling, and the effect was lost in PlGF knockout mice. ${ }^{34)}$ PlGF may be a novel downstream target of vascular MR that mediates aldos- terone-related angiogenesis in failing hearts, as well as in vascular remodeling. These findings suggest a nonrenal mechanism for the vascular protective effects of aldosterone antagonists in humans and supports targeting the vascular aldosterone/MR/PlGF/Flt-1 pathway as a therapeutic strategy for ischemic heart failure and cardiac remodeling. Discerning which of the effects of sFlt-1 is responsible for our observed clinical associations will require additional laboratory work.

Limitations: The major strength of our study is that it is the first to describe a prospective association between sFlt-1 and BNP levels. However, several limitations of the study also need to be noted. First, although we found a significant relationship between sFlt-1 and BNP elevation, the size of the additive impact on other risk factors remains to be elucidated, because the associations shown in our study were not very strong. Second, because this was an observational study, assessment of the biomarkers, sFlt-1 and PIGF, occurred only on 1 occasion. Including an analysis of the serial changes in biomarker levels might have made our results more clinically useful. Third, many of our study subjects were on multiple medications, and we could not completely match patient backgrounds. The use of propensity score analysis may be a valuable option in a larger clinical study; if the sample size was larger and the follow-up period was longer, we may have obtained statistical significance for some important variables.

Conclusions: The present 5-year follow-up study demonstrated that circulating levels of sFlt-1 were increased in patients with CAD, and high sFlt-1 levels in patients with CAD were indicative of a moderate increase in BNP levels. Moreover, $\triangle \mathrm{BNP}$ was correlated with $\triangle \mathrm{ESVI} /$ year in CAD patients with high sFlt-1 baseline levels. These data suggest that a high sFlt1 level may be an effective biomarker for predicting the progression of heart failure in patients with CAD.

\section{Disclosure}

This work was partly presented at the American Heart Association 2011 Scientific Sessions in Orlando, FL, USA.

\section{REFERENCES}

1. Mudd JO, Kass DA. Tackling heart failure in the twenty-first century. Nature 2008; 451: 919-28. (Review)

2. Ock S, Ahn J, Lee SH, et al. Receptor activator of nuclear factorkappaB ligand is a novel inducer of myocardial inflammation. Cardiovasc Res 2012; 94: 105-14.

3. Eaton LW, Weiss JL, Bulkley BH, Garrison JB, Weisfeldt ML. Regional cardiac dilatation after acute myocardial infarction: recognition by two-dimensional echocardiography. N Engl J Med 1979; 300: 57-62.

4. Erlebacher JA, Weiss JL, Eaton LW, Kallman C, Weisfeldt ML, Bulkley BH. Late effects of acute infarct dilation on heart size: a two dimensional echocardiographic study. Am J Cardiol 1982; 49: 1120-6.

5. McKay RG, Pfeffer MA, Pasternak RC, et al. Left ventricular remodeling after myocardial infarction: a corollary to infarct expansion. Circulation 1986; 74: 693-702.

6. Konstam MA, Kramer DG, Patel AR, Maron MS, Udelson JE. Left ventricular remodeling in heart failure: current concepts in clinical significance and assessment. JACC Cardiovasc Imaging 2011; 4: 98-108. (Review) 
7. Shibuya M. Vascular endothelial growth factor receptor-1 (VEGFR-1/Flt-1): a dual regulator for angiogenesis. Angiogenesis 2006; 9: 225-30. (Review)

8. Khurana R, Moons L, Shafi S, et al. Placental growth factor promotes atherosclerotic intimal thickening and macrophage accumulation. Circulation 2005; 111: 2828-36.

9. Kendall RL, Wang G, Thomas KA. Identification of a natural soluble form of the vascular endothelial growth factor receptor, FLT1, and its heterodimerization with KDR. Biochem Biophys Res Commun 1996; 226: 324-8.

10. Maynard SE, Min JY, Merchan J, et al. Excess placental soluble fms-like tyrosine kinase 1 (sFlt1) may contribute to endothelial dysfunction, hypertension, and proteinuria in preeclampsia. J Clin Invest 2003; 111: 649-58.

11. Onoue K, Uemura S, Takeda Y, et al. Reduction of circulating soluble fms-like tyrosine kinase-1 plays a significant role in renal dysfunction-associated aggravation of atherosclerosis. Circulation 2009; 120: 2470-7.

12. Heeschen C, Dimmeler S, Fichtlscherer S, et al. Prognostic value of placental growth factor in patients with acute chest pain. JAMA 2004; 291: 435-41.

13. Iwama H, Uemura S, Naya N, et al. Cardiac expression of placental growth factor predicts the improvement of chronic phase left ventricular function in patients with acute myocardial infarction. Am Coll Cardiol 2006; 47: 1559-67.

14. Lenderink T, Heeschen C, Fichtlscherer S, et al. Elevated placental growth factor levels are associated with adverse outcomes at fouryear follow-up in patients with acute coronary syndromes. Am Coll Cardiol 2006; 47: 307-11.

15. Onoue K, Umemura S, Takeda Y, et al. Usefulness of soluble Fmslike tyrosine kinase-1 as a biomarker of acute severe heart failure in patients with acute myocardial infarction. Am J Cardiol 2009; 104: 1478-83.

16. Kodama Y, Kitta Y, Nakamura T, et al. Atorvastatin increases plasma soluble Fms-like tyrosine kinase-1 and decreases vascular endothelial growth factor and placental growth factor in association with improvement of ventricular function in acute myocardial infarction. Am Coll Cardiol 2006; 48: 43-50.

17. Belgore FM, Blann AD, Lip GY. sFlt-1, a potential antagonist for exogenous VEGF. Circulation 2000; 102: E108-9.

18. Nakamura T, Funayama H, Kubo N, et al. Elevation of plasma placental growth factor in the patients with ischemic cardiomyopathy. Int J Cardiol 2009; 131: 186-91.

19. Omland T, Aakvaag A, Vik-Mo H. Plasma cardiac natriuretic peptide determination as a screening test for the detection of patients with mild left ventricular impairment. Heart 1996; 76: 232-7.

20. Davidson NC, Naas AA, Hanson JK, Kennedy NS, Coutie WJ, Struthers AD. Comparison of atrial natriuretic peptide B-type natriuretic peptide, and $\mathrm{N}$-terminal proatrial natriuretic peptide as indicators of left ventricular systolic dysfunction. Am J Cardiol 1996; 77: 828-31.

21. Mukoyama M, Nakao K, Saito Y, et al. Increased human brain natriuretic peptide in congestive heart failure. N Engl J Med 1990; 323: 757-8.

22. Tsutamoto T, Wada A, Maeda K, et al. Attenuation of compensation of endogenous cardiac natriuretic peptide system in chronic heart failure: prognostic role of plasma brain natriuretic peptide concentration in patients with chronic symptomatic left ventricular dysfunction. Circulation 1997; 96: 509-16.

23. Nishigaki K, Tomita M, Kagawa K, et al. Marked expression of plasma brain natriuretic peptide is a special feature of hypertrophic obstructive cardiomyopathy. Am Coll Cardiol 1996; 28: 1234-42.

24. Nakamura M, Endo H, Nasu M, Arakawa N, Segawa T, Hiramori $\mathrm{K}$. Value of plasma B type natriuretic peptide measurement for heart disease screening in a Japanese population. Heart 2002; 87: 131-5.

25. Belgore FM, Blann AD, Li-Saw-Hee FL, Beevers DG, Lip GY. Plasma levels of vascular endothelial growth factor and its soluble receptor (SFlt-1) in essential hypertension. Am J Cardiol 2001; 87: 805-7, A9.

26. Walsh K, Shiojima I. Cardiac growth and angiogenesis coordinated by intertissue interactions. J Clin Invest 2007; 117: 3176-9.

27. Izumiya Y, Shiojima I, Sato K, Sawyer DB, Colucci WS, Walsh K. Vascular endothelial growth factor blockade promotes the transition from compensatory cardiac hypertrophy to failure in response to pressure overload. Hypertension 2006; 47: 887-93.

28. Giordano FJ, Gerber HP, Williams SP, et al. A cardiac myocyte vascular endothelial growth factor paracrine pathway is required to maintain cardiac function. Proc Natl Acad Sci USA 2001; 98: 5780-5.

29. Di Marco GS, Reuter S, Hillebrand U, et al. The soluble VEGF receptor sFlt1 contributes to endothelial dysfunction in CKD. J Am Soc Nephrol 2009; 20: 2235-45.

30. Levine RJ, Maynard SE, Qian C, et al. Circulating angiogenic factors and the risk of preeclampsia. N Engl J Med 2004; 350: 67283.

31. Rogerson FM, Fuller PJ. Mineralocorticoid action. Steroids 2000; 65: 61-73. (Review)

32. Pitt B, Zannad F, Remme WJ, et al. The effect of spironolactone on morbidity and mortality in patients with severe heart failure. Randomized Aldactone Evaluation Study Investigators. N Engl J Med 1999; 341: 709-17.

33. Pitt B, Remme W, Zannad F, et al. Eplerenone, a selective aldosterone blocker, in patients with left ventricular dysfunction after myocardial infarction. N Engl J Med 2003; 348: 1309-21.

34. Jaffe IZ, Newfell BG, Aronovitz M, et al. Placental growth factor mediates aldosterone-dependent vascular injury in mice. J Clin Invest 2010; 120: 3891-900 